Pädiatrie und Pädologie 2013 · 48:5

DOI 10.1007/s00608-013-0115-1

C) Springer-Verlag Wien 2013

\section{E. Tatzer}

Sprecher des Vereins "Politische Kindermedizin", Hinterbrühl

\title{
Partizipation - ein Thema in der Kindermedizin ?
}

Kindermediziner und Eltern sind gewöhnt, für Kinder und Jugendliche $\mathrm{zu}$ sprechen. Im Bewusstsein, die Anliegen und Bedürfnisse der Kinder und Jugendlichen - insbesondere im Hinblick auf deren Zukunft - gut oder sogar besser als diese selbst zu kennen, tendieren sie im Allgemeinen dazu, Entscheidungen auch über deren Kopf hinweg zu treffen. Entscheidungsteilhabe als wichtige Gesundheitsressource ist heute weitgehend anerkannt und sollte auch in der Kindermedizin ihren Platz finden. Partizipation bedeutet aber auch Teilhabe am gesellschaftlichen Leben, und es stellt sich die Frage, wie weit medizinische Maßnahmen Kindern und Jugendlichen dazu verhelfen können.

Die 7. Jahrestagung der Politischen Kindermedizin vom 15.-16. November 2013 in Salzburg mit dem Titel „Partizipation in der Kindermedizin- von der Versorgung zur Teilhabe“ soll ein bisher eher vernachlässigtes Gebiet der Pädiatrie thematisieren.

Dabei werden sich Dr. Simon LENTON, Bath, UK, , Prof. Dr. Jochen H.H. EHRICH, Hannover, Deutschland, und Hanne SIEBER, Schweiz, mit den „Internationalen Sichtweisen“" aus- einandersetzen. Weitere Themenblöcke sind Entscheidungsteilhabe von Kindern und Jugendlichen in der Praxis - mit Berichten von bereits existierenden Modellen und Beiträgen aus und für die Praxis in Österreich - und Kinder- und Jugendmedizin als Hilfe zur Teilhabe, sowie ein Round Table Partizipation von Kindern und Jugendlichen in der Medizin wer, was, wann, warum, wozu ? unter der Leitung von Prof. Reinhold KERBL (Präsident der ÖGKJ). Darüber hinaus wird heuer wieder ausreichend Zeit für Diskussionen und die produktiven Treffen unserer permanenten Arbeitsgruppen sein. Wir erwarten eine informative und spannende Tagung zu einem innovativen Thema.

\section{) Die 7. Jahrestagung der Politischen Kindermedizin vom 15.-16. November 2013 in Salzburg soll ein bisher eher vernachlässigtes Gebiet der Pädiatrie thematisieren.}

Partizipation - im Sinne der Teilhabe an der Zivilgesellschaft ist auch der Grundgedanke der Politischen Kindermedizin. 2007 hat es mit der Veranstaltung der ersten Jahrestagung „Kindermedizin - Werte versus Ökonomie" durch eine kleine, aber bundesweit organisierte Initiativgruppe begonnen. Waren wir damals noch ganz unsicher, ob Kindermediziner an einer pointiert kritischen Auseinandersetzung überhaupt Interesse finden werden, ist unsere Plattform nunmehr zu einem konstanten Faktor in der Diskussion zur Versorgung von Kindern und Jugendlichen in unserem Land geworden. Zunehmend werden unsere Diskussionsbeiträge in ihrer konstruktiven Form auch von offiziellen Stellen anerkannt und wir, die Politische Kindermedizin bzw. einzelne Vertreter zur Mitarbeit in verschiedenen Gremien bzw. Projekten eingeladen. Dies übersteigt zunehmend die Möglichkeiten einer rein informellen Plattform, und wir haben uns daher zur Gründung des Vereins „Politische Kindermedizin“ entschlossen.

Jenseits jeglicher „Vereinsmeierei“ wollen wir auch in Zukunft unsere ursprüngliche Idee der kritischen Auseinandersetzung mit den bestehenden Verhältnissen hochhalten. Den Weg dazu sehen wir weiterhin im Aufgreifen brisanter Themen, in der Erstellung gediegener Analysen, ihrer Verbreitung und Bekanntmachung in der Öffentlichkeit, sowie in der Erarbeitung bzw. Miterarbeitung neuer Wege. Der Verein steht alle Menschen offen, die helfen wollen, die Anliegen der Kinder auf medizinischem Gebiet mit politischen Mitteln durchzusetzen.

Die erste Generalversammlung des neuen Vereins findet im Anschluss an die Jahrestagung am 16.11.2013 statt und ist offen für alle Interessierten und präsumtiven Vereinsmitglieder. Dazu laden wir herzlich ein.

\section{Dr. Ernst Tatzer}

Sprecher

Verein "Politische Kindermedizin"

Internet: http://www.polkm.org

E-Mail: ernst.tatzer@kabsi.at 